\title{
Erratum: Mosquito (Diptera: Culicidae) fauna in parks in greater São Paulo, Brazil
}

No artigo "Mosquito (Diptera: Culicidae) fauna in parks in greater São Paulo, Brazil" publicado do periódico Biota Neotropica, 15(3), na página 1 onde lia-se:

Marcia Bicudo de Paula ${ }^{I}$, Aristides Fernandes ${ }^{I}$, Antônio Ralph Medeiros-Sousa ${ }^{I}$, Walter Ceretti-Júnior ${ }^{1}$, Rafael Christe $^{I}$, Regina Claudia Stroebel ${ }^{2}$, Leila Pedrosa ${ }^{2}$, Rosa Maria Marques de Sá Almeida ${ }^{1}$, Uellinton Damasceno Pereira ${ }^{3}$, Marcelo Cassiano de Oliveira Jacintho ${ }^{3}$, Delsio Natal ${ }^{1}$, Mauro Toledo Marrelli ${ }^{14}$

Leia-se:

Marcia Bicudo de Paula ${ }^{I}$, Aristides Fernandes ${ }^{I}$, Antônio Ralph Medeiros-Sousa ${ }^{1}$, Walter Ceretti-Júnior ${ }^{I}$, Rafael $^{\prime}$ Christe $^{I}$, Regina Claudia Stroebel ${ }^{2}$, Leila Pedrosa ${ }^{2}$, Rosa Maria Marques de Sá Almeida ${ }^{1}$, Gabriela Cristina de Carvalho ${ }^{1}$, Uellinton Damasceno Pereira ${ }^{3}$, Marcelo Cassiano de Oliveira Jacintho ${ }^{3}$, Delsio Natal, Mauro Toledo Marrelli ${ }^{14}$ 